\title{
VLTI/AMBER observations of the Seyfert nucleus of NGC $3783^{\star}$
}

\author{
G. Weigelt ${ }^{1}$, K.-H. Hofmann ${ }^{1}$, M. Kishimoto ${ }^{1}$, S. Hönig ${ }^{2}$, D. Schertl ${ }^{1}$, A. Marconi ${ }^{3,4}$, F. Millour ${ }^{5}$, R. Petrov ${ }^{5}$, \\ D. Fraix-Burnet ${ }^{6}$, F. Malbet ${ }^{6}$, K. Tristram ${ }^{1}$, and M. Vannier ${ }^{5}$ \\ ${ }^{1}$ Max-Planck-Institut für Radioastronomie, Auf dem Hügel 69, 53121 Bonn, Germany \\ e-mail: weigelt@mpifr.de \\ 2 UCSB Department of Physics, Broida Hall 2015H, Santa Barbara CA, USA \\ 3 Dipartimento di Fisica e Astronomia, Universitá di Firenze, Largo Enrico Fermi 2, 510125 Firenze, Italy \\ ${ }^{4}$ INAF - Osservatorio Astrofisico di Arcetri, Largo Fermi 5, 50125 Firenze, Italy \\ 5 Laboratoire Lagrange, UMR 7293, Université de Nice Sophia-Antipolis, CNRS, Observatoire de la Côte d'Azur, 06300 Nice, \\ France \\ ${ }^{6}$ Université Joseph Fourier (UJF) - Grenoble 1/CNRS-INSU, Institut de Planétologie et d'Astrophysique de Grenoble (IPAG) \\ UMR 5274, 38041 Grenoble, France
}

Received 13 March 2012 / Accepted 6 April 2012

\section{ABSTRACT}

\begin{abstract}
Context. The putative tori surrounding the accretion disks of active galactic nuclei (AGNs) play a fundamental role in the unification scheme of AGNs. Infrared long-baseline interferometry allows us to study the inner dust distribution in AGNs with unprecedented spatial resolution over a wide infrared wavelength range.

Aims. Near- and mid-infrared interferometry is used to investigate the milli-arcsecond-scale dust distribution in the type 1.5 Seyfert nucleus of NGC 3783.

Methods. We observed NGC 3783 with the VLTI/AMBER instrument in the $K$-band and compared our observations with models. Results. From the $K$-band observations, we derive a ring-fit torus radius of $0.74 \pm 0.23$ mas or $0.16 \pm 0.05$ pc. We compare this size with infrared interferometric observations of other AGNs and UV/optical-infrared reverberation measurements. For the interpretation of our observations, we simultaneously model our near- and mid-infrared visibilities and the spectral energy distribution (SED) with a temperature/density-gradient model including an additional inner hot $1400 \mathrm{~K}$ ring component.
\end{abstract}

Key words. galaxies: active - galaxies: Seyfert - techniques: interferometric - galaxies: individual: NGC 3783

\section{Introduction}

The putative tori surrounding the accretion disks of active galactic nuclei (AGNs) play a fundamental role in the unification scheme of AGNs (Antonucci 1993). These tori most likely provide the material reservoir that feeds the accretion disk (e.g., Krolik \& Begelman 1988). Infrared interferometric observations can resolve these mas-scale tori in the near-infrared (NIR) (e.g., Swain et al. 2003; Wittkowski et al. 2004; Kishimoto et al. 2009a; Pott et al. 2010; Kishimoto et al. 2011a) and mid-infrared (MIR) (e.g., Jaffe et al. 2004; Meisenheimer et al. 2007; Tristram et al. 2007, 2009; Beckert et al. 2008; Kishimoto et al. 2009b; Raban et al. 2009; Burtscher et al. 2009, 2010; Tristram \& Schartmann 2011; and Kishimoto et al. 2011b, Paper I). In this paper, we present the first NIR interferometric observation of an AGN with the AMBER/VLTI instrument.

\section{Observations and data reduction}

We observed the Seyfert 1.5 AGN NGC 3783 in 2009 and 2011 (IDs 083.B-0212 and 087.B-0578) with the ESO VLTI and the AMBER instrument (Petrov et al. 2007). For these observations in the $K$-band (see Table 1 ), the AMBER low spectral resolution mode (LR) was employed. Figure 1 (top) presents examples of target interferograms to illustrate the noise problem $(K \sim 10.1)$. Long detector integration times (DIT) of 400 and

* Based on observations made with ESO telescopes at Paranal Observatory under programme IDs 083.B-0212(A) and 087.B-0578(A).
$800 \mathrm{~ms}$ were chosen to be able to recognize the faint fringes during data recording and correct drifts of the optical path differences (OPDs) between the telescope beams. The interferograms shown in Fig. 1 are two-telescope interferograms, not AMBERstandard three-telescope interferograms. In 2009 (see Table 1), we recorded two-telescope interferograms since it is easier to correct OPD drifts in two-telescope than in three-telescope interferograms. In 2011, we recorded three-telescope interferograms, which provide closure phases. For data reduction of the two-telescope interferograms, we used our own software (developed by one of us, $\mathrm{KHH}$ ), which is able to reduce the nonstandard two-telescope interferograms. It is based on the same P2VM algorithm (Tatulli et al. 2007; Chelli et al. 2009) as the AMBER $a m d l i b{ }^{1}$ software. To reduce the effect of the instantaneous OPDs on the visibility, we applied a preprocessing method that equalizes the OPD histograms of the target and calibrator interferograms (Kreplin et al. 2012). Figure 1 and Table 1 show the derived visibilities, which are wavelength averages over the wavelength range of $2.0-2.40 \mu \mathrm{m}$. For data reduction of the 2011 three-telescope data, we used the standard AMBER data reduction package amdlib version 3.0. Figure 1 (bottom) shows the closure phases of NGC 3783 derived from the three-telescope 2011 interferograms. The average closure phase is $3.3 \pm 26^{\circ}$. Closure phases are a measure of asymmetry. However, the large errors do not allow us to detect any asymmetry. We also tried

\footnotetext{
1 The AMBER - reduction package amdlib is available at: http://www.jmmc.fr/data_processing_amber.htm
} 
Table 1. Observation log of our AMBER LR observations of NGC 3783 and its calibrator CD-37 7391.

\begin{tabular}{|c|c|c|c|c|c|c|c|c|}
\hline Name & Date & $\begin{array}{c}\text { Time of } \\
\text { observation } \\
\text { (UTC) }\end{array}$ & $\begin{array}{l}\text { Telescopes/ } \\
\text { proj. baseline } \\
\text { lengths }(\mathrm{m})\end{array}$ & $\begin{array}{l}\text { PA } \\
\left({ }^{\circ}\right)\end{array}$ & $\begin{array}{l}\text { DIT } \\
(\mathrm{ms})\end{array}$ & $\begin{array}{c}\text { Seeing } \\
(\operatorname{arcsec})\end{array}$ & $\begin{array}{l}\text { Number } \\
\text { of frames }\end{array}$ & $\begin{array}{c}\text { Target } \\
\text { visibility }\end{array}$ \\
\hline NGC 3783 & $09 / 04 / 14$ & $01: 19-01: 31$ & UT2-3/46.6 & 28.8 & 800 & 0.93 & $11 \times 70$ & 0.89 \\
\hline CD-37 7391 & $09 / 04 / 14$ & $01: 45-01: 55$ & UT2-3 & & 800 & 0.84 & 10 & \\
\hline NGC 3783 & $09 / 04 / 14$ & $03: 16-03: 29$ & UT2-3/45.2 & 44.8 & 800 & 0.82 & $11 \times 70$ & 0.96 \\
\hline NGC 3783 & $09 / 04 / 14$ & $03: 32-03: 41$ & UT2-3/44.8 & 46.3 & 400 & 0.79 & $10 \times 70$ & 0.93 \\
\hline CD-37 7391 & $09 / 04 / 14$ & $04: 31-04: 34$ & UT2-3 & & 400 & 0.78 & $4 \times 120$ & \\
\hline NGC 3783 & $09 / 04 / 14$ & $03: 52-04: 03$ & UT3-4/62.0 & 120.9 & 800 & 0.68 & $10 \times 70$ & 0.97 \\
\hline CD-37 7391 & $09 / 04 / 14$ & $02: 11-02: 16$ & UT3-4 & & 800 & 1.45 & $5 \times 70$ & \\
\hline CD-37 7391 & $09 / 04 / 14$ & $02: 00-02: 05$ & UT2-4 & & 800 & 0.99 & $5 \times 120$ & \\
\hline NGC 3783 & $09 / 04 / 14$ & $03: 44-03: 49$ & UT2-4/87.1 & 90.0 & 800 & 0.72 & $5 \times 70$ & 0.86 \\
\hline NGC 3783 & $09 / 04 / 14$ & 04:12-04:20 & UT2-4/84.6 & 94.7 & 800 & 0.81 & $8 \times 70$ & 0.85 \\
\hline CD-37 7391 & $09 / 04 / 14$ & $04: 41-04: 45$ & UT2-4 & & 800 & 0.87 & $3 \times 120$ & \\
\hline NGC 3783 & $11 / 05 / 18$ & $02: 39-02: 44$ & UT1-2-4/51.2/113.8/80.3 & $38.8 / 77.3 / 100.7$ & 400 & 0.63 & $7 \times 120$ & \\
\hline NGC 3783 & $11 / 05 / 18$ & $02: 50-02: 55$ & UT1-2-4/50.6/111.2/78.7 & $39.8 / 78.9 / 102.8$ & 400 & 0.68 & $7 \times 120$ & \\
\hline CD-37 7391 & $11 / 05 / 18$ & 02:00-02:06 & UT1-2-4/ & & 400 & 0.75 & $7 \times 120$ & \\
\hline CD-37 7391 & $11 / 05 / 18$ & $02: 09-02: 17$ & UT1-2-4/ & & 400 & 0.63 & $7 \times 120$ & \\
\hline
\end{tabular}

Notes. The data in the first 11 lines were observed in the two-telescope mode and the data in the last 4 lines in the three-telescope mode. The table lists the names, times of observations, projected baseline lengths, position angles PA, detector integration times DIT, seeing, number of interferograms, and derived target visibilities (errors \pm 0.09 ).

Table 2. NGC 3783 torus radius $R_{\text {torus }}$, 2MASS fluxes of the nuclear core, and flux contributions of the host galaxy and the AD.

\begin{tabular}{lcccccccccc}
\hline \hline $\begin{array}{l}J \text { flux } \\
(\mathrm{mJy})\end{array}$ & $\begin{array}{c}H \text { flux } \\
(\mathrm{mJy})\end{array}$ & $\begin{array}{c}K \text { flux } \\
(\mathrm{mJy})\end{array}$ & $\begin{array}{c}J \\
(\mathrm{mag})\end{array}$ & $\begin{array}{c}H \\
(\mathrm{mag})\end{array}$ & $\begin{array}{c}K \\
(\mathrm{mag})\end{array}$ & $\begin{array}{c}\text { Host } \\
\text { fraction }^{a}\end{array}$ & $\begin{array}{c}\mathrm{AD} \\
\text { fraction }^{b}\end{array}$ & $\begin{array}{c}R_{\text {torus }} \\
(\mathrm{mas})\end{array}$ & $\begin{array}{c}R_{\text {torus }} \\
(\mathrm{pc})\end{array}$ & $\begin{array}{c}R_{\tau_{\mathrm{K}}}{ }^{c} \\
(\mathrm{pc})\end{array}$ \\
\hline 18.8 & 34.2 & 61.8 & 12.3 & 11.2 & 10.1 & $0.005 \pm 0.002$ & $0.21 \pm 0.07$ & $0.74 \pm 0.23$ & $0.16 \pm 0.05$ & $0.071 \pm 0.025$ \\
\hline
\end{tabular}

Notes. ${ }^{(a)} K$-band flux contribution in the AMBER FOV. ${ }^{(b)}$ AD flux contribution to the point-like core in the 2 MASS $K$-band image. (c) Reverberation radius $R_{\tau_{\mathrm{K}}}$ (Glass 1992).

to derive calibrated visibilities from the 2011 data, but without success since the transfer function was unstable.

\section{Geometric model fits}

To interpret our $K$-band visibilities (Fig. 1 middle), we first fitted a geometric thin-ring model (i.e., ring width = outer radius minus inner radius $=0$ ) to the visibilities and derived a ring-fit radius of $\sim 0.67$ mas. This is only a very rough estimate of the torus size since the observed visibilities may not only depend on the torus but also on the underlying galaxy within the 60 mas field-of-view (FOV) of AMBER and on the accretion disk (AD), which is thought to remain unresolved (Kishimoto et al. 2007, 2009a,b). Therefore, we have to estimate the flux contributions from the host galaxy and the AD point source and take these contributions into account when fitting the visibilities.

From the $K$-band image of NGC 3783 in the 2MASS cata$\log$, we estimated the flux contribution of the host galaxy within the 60 mas AMBER FOV to $0.5 \pm 0.2 \%$. To obtain the NIR flux from the torus and the AD, we used two-dimensional fits to separate the point-like core component in the 2MASS $J_{-}, H$-, and $K$-band images from the underlying host galaxy (see Table 2 ), following the same procedure as described by Kishimoto et al. (2009a). Using the derived NIR core fluxes, we can estimate the flux contribution of the AD component in the $K$ band. We assume here that the core component flux originates from the hot dust and from the AD. Therefore, we fitted a power-law spectrum for the AD and a blackbody for the dust emission, as described in Kishimoto et al. (2009a). We also applied a small correction for Galactic reddening with $E_{B-V}=0.119$. By assigning an uncertainty of the NIR AD spectral index of 0.3 , we also obtained the uncertainty of the $K$-band AD flux contribution. The
AD flux fraction in the $K$-band was estimated to be as small as $21 \pm 7 \%$, which is similar to the values of several other AGNs reported by Kishimoto et al. (2007, 2009a). If we now take into account these estimated flux contributions of $\sim 21 \%$ from the unresolved $\mathrm{AD}$ and of $\sim 0.5 \%$ from the host galaxy, we can derive the visibilities of the torus itself and can fit the radius of the torus (i.e., this radius is the only fit parameter; the AD contributes just a constant of 0.21 to the total visibilities). We derive a torus radius $R_{\text {torus }}$ of $0.74 \pm 0.23$ mas or $0.16 \pm 0.05$ pc (thin-ring fit; see Fig. 1 middle, red curve).

\section{Interpretation and discussion}

\subsection{NIR interferometric and reverberation radii}

Figure 2 compares the derived ring-fit radius $R_{\text {torus }} \sim 0.16 \mathrm{pc}$ of NGC 3783 (red) with eight interferometric $K$-band radii (blue) reported by Kishimoto et al. (2009a, 2011a). These radii are plotted against the UV luminosity $L$, defined as a scaled $V$-band luminosity of $6 v f_{v}(V)$, with the $V$ flux extrapolated from the flux at $1.2 \mu \mathrm{m}$ (Kishimoto et al. 2007). We can compare these torus radii with reverberation radii $R_{\tau_{\mathrm{K}}}$ (black) derived from the light traveling distances corresponding to the time lag between the $K$-band and the UV/optical (Suganuma et al. 2006). They are known to be proportional to $\sim L^{1 / 2}$ and are likely probing the dust sublimation radius. The dotted line is the fit curve of the reverberation radii (different luminosity values are obtained for the same object because of variability and uncertainties of the luminosity derivation). The reverberation radius of NGC 3783 is $\sim 0.071 \mathrm{pc}$, which is smaller than the interferometric torus radius $R_{\text {torus }} \sim 0.16 \mathrm{pc}$ (Sect. 3). Figure 2 shows that several interferometric torus radii are larger than the reverberation radii. Our interpretation is that the interferometric torus radii are averages 

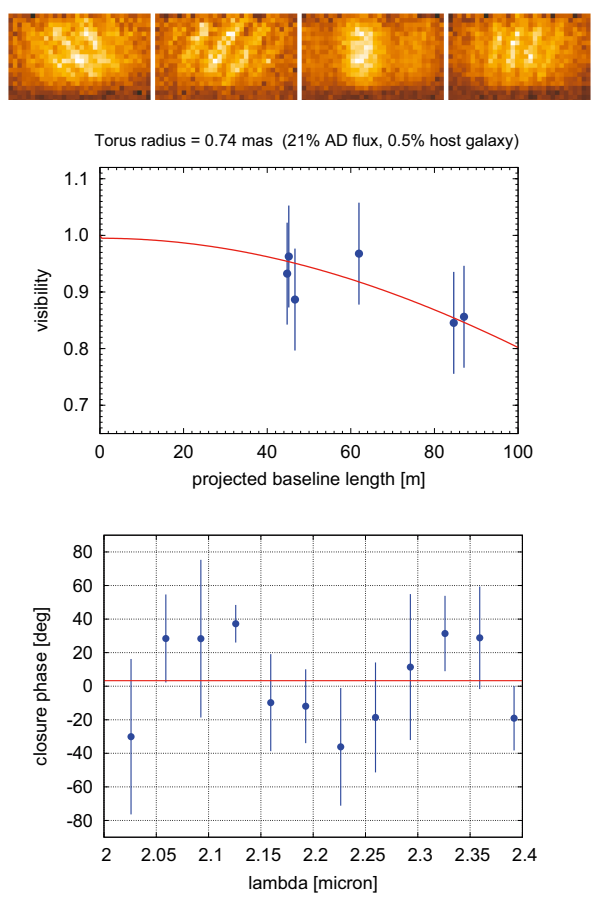

Fig. 1. Top: examples of LR $K$-band AMBER interferograms of NGC 3783 ( 2.0-2.4 $\mu$ m from bottom to top), which illustrate the noise problem. From left to right, the first and second interferogram are recorded with UT2-UT3 (46.6 m projected baseline) with DIT $=800 \mathrm{~ms}$ and $400 \mathrm{~ms}$, respectively, the third one with UT3-UT4 $(62.5 \mathrm{~m}, 800 \mathrm{~ms})$, and the last one with UT2-UT4 $(89.4 \mathrm{~m}, 800 \mathrm{~ms})$. Middle: calibrated visibilities of NGC 3783 and geometric ring-model fit (red). We derive a ring-fit torus radius of $0.74 \pm 0.23$ mas or $0.16 \pm 0.05$ pc. Bottom: closure phases plotted versus wavelength.

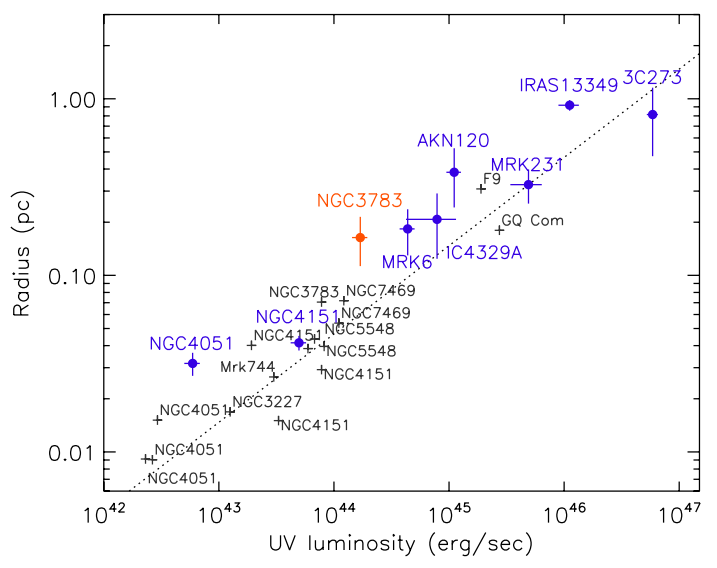

Fig. 2. $K$-band torus radii of NGC 3783 (red dot) and eight other AGNs (blue; from Kishimoto et al. 2011a) versus their UV luminosities. The black symbols and the dotted line are the reverberation radii $R_{\tau_{\mathrm{K}}}$ (Glass 1992; Suganuma et al. 2006) and their fit curve, respectively.

over the radial dust distribution that emits the $K$-band light, whereas the reverberation radii probably trace the dust closer to the inner dust torus boundary radius (Kishimoto et al. 2009a). Furthermore, we note that $R_{\text {torus }} \sim 0.16$ pc is a fit radius calculated with a thin-ring model (i.e., ring width $=$ outer radius minus inner radius $=0$ ). If a dust distribution with a certain thin-ring fit radius is ring-like and has a ring width larger than zero, then the inner ring radius would be smaller than the thin-ring fit radius. We have not fitted a ring model with a larger ring width, since the ring width cannot be constrained with the available visibilities.

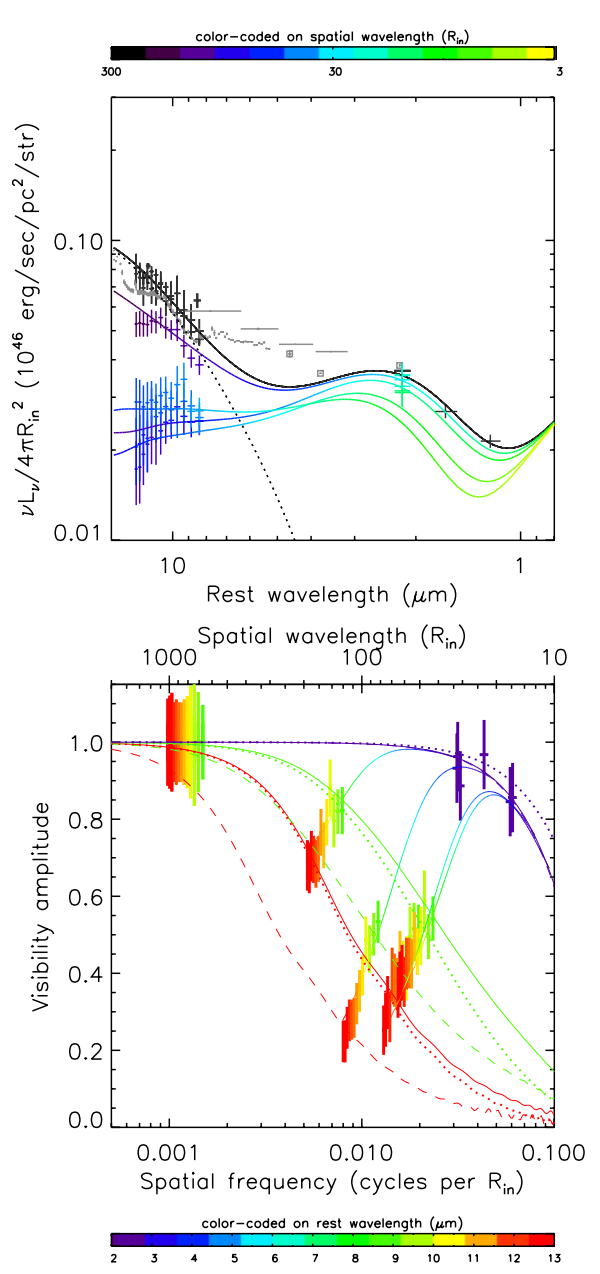

Fig. 3. Temperature/density-gradient model including an additional inner hot $1400 \mathrm{~K}$ ring component. Top: SED observations (black and gray symbols), model SEDs (black solid line: model including the $1400 \mathrm{~K}$ ring component; black dotted line: model without the $1400 \mathrm{~K}$ component), and correlated fluxes (yellow, green, and blue; see Paper I for more details). The different colors (see top color bar) correspond to different spatial wavelengths measured in units of the dust sublimation radius $R_{\text {in }}$. Bottom: new NIR visibilities (purple symbols) and our MIR visibilities from Paper I (the symbols with colors from green to red correspond to 8.5 to $13 \mu \mathrm{m}$; see color-coding bar; note that the spatial frequency is given in units of cycles per $R_{\text {in }}$ ). The solid and dashed lines are the visibilities of the temperature/density-gradient model including an additional inner $1400 \mathrm{~K}$ ring. The red, green, and purple lines are the model visibilities at $13,8.5$, and $2.2 \mu \mathrm{m}$, respectively (solid lines: model curves for the PA along the equatorial axis; dashed line: along the polar direction; see Paper I). The dotted lines are the visibilities and SED of the same temperature/density-gradient model, but without an inner hot $1400 \mathrm{~K}$ ring component.

\subsection{Simultaneous modeling of the NIR AMBER visibilities, the MIR MIDI visibilities, and the SED}

Mid-infrared (MIR) MIDI interferometry of NGC 3783 was reported by Beckert et al. (2008), Kishimoto et al. (2009b), and Kishimoto et al. (2011b) (=Paper I). For the interpretation of these observations, we used a temperature/density-gradient model including an additional hot inner ring component with a temperature of $1400 \mathrm{~K}$ (Paper I). This simple model assumes that the face-on surface brightness distribution of the torus is dominated by the IR radiation from dust clouds directly illuminated and heated by the AD. These dust clouds are probably located 
near the torus surface since clouds deep inside the torus are not directly illuminated.

The surface brightness distribution of this temperature/ density-gradient model depends on two distributions, namely a radial temperature and a radial surface density distribution (see Eq. (8) in Paper I). The maximum dust temperature $T_{\max }(r)$ at distance $r$ is assumed to be proportional to $\left(r / R_{\text {in }}\right)^{\beta}$, where $r$ is the radial distance, $\beta$ is the power-law index, and $R_{\text {in }}$ is the dust sublimation radius empirically given by the NIR reverberation radius $R_{\tau_{\mathrm{K}}}$ (Glass 1992), i.e., we define $R_{\text {in }}=R_{\tau_{\mathrm{K}}}$ (Paper I, Eq. (1)).

Furthermore, the surface brightness distribution depends on the surface density function $f_{\epsilon}(r)=f_{0}\left(r / R_{\text {in }}\right)^{\alpha}$ of the heated dust clouds near the surface (power law with index $\alpha$ ). The emissivity factor $f_{0}$ is equal to $f_{\epsilon}(r)$ at $r$ equal to the sublimation radius $R_{\text {in }}$. Our IR observations are only sensitive to the dust clouds near the surface, which have the temperature $T_{\max }(r)$, and not to the cold dust inside the torus. $f_{\epsilon}(r)$ can be regarded as a surface filling factor multiplied by the emissivity (Paper I). If the emissivity of optically thick illuminated clouds does not depend sensitively on the radial distance from the illuminating source or the observing wavelength (e.g., see Fig. 3 of Hönig \& Kishimoto 2010), the factor $f_{\epsilon}(r)$ is roughly proportional to the radial surface density distribution of the heated dust.

Interestingly, the application of this temperature/densitygradient model to several AGNs in Paper I and to the NGC 3783 observations reported in this paper (see Fig. 3) has shown that an additional inner hot model component is required with a temperature of $1400 \mathrm{~K}$ and a radius of one or a few dust sublimation radii in order to explain all observations. This hot component might play a similar role as the puffed-up inner rim discovered in several young stellar objects near the dust sublimation radius.

We simultaneously fitted this temperature/density-gradient model including an inner $1400 \mathrm{~K}$ ring to our new $K$-band data as well as the MIR data and the SED from Paper I. The goal of this modeling is to further constrain physical parameters of the dust distribution. Figure 3 shows that this model is able to simultaneously reproduce all NIR and MIR visibilities as well as the SED. In Fig. 3 (bottom), the NIR visibilities (purple) and the MIR visibilities are shown (from green to red, wavelengths 8.5 to $13 \mu \mathrm{m}$; see color-coding bar). The purple, red, and green curves are the model visibilities at $2.2,13$, and $8.5 \mu \mathrm{m}$, respectively. The solid lines are the model curves along the PA of the equatorial axis, the dashed line along the polar direction, as defined by optical polarization measurements (see Paper I, where this elliptical and a circular symmetric model are presented).

If there is no hot inner ring component added to the above temperature/density-gradient model, then the $K$-band model visibilities (blue dotted line in Fig. 3, bottom) are systematically higher than observed and the model SED (black dotted line in Fig. 3, top) has a deficiency in the NIR. Therefore, the above inner $1400 \mathrm{~K}$ ring component is required to explain the data.

This new modeling including $K$-band visibilities (Fig. 3 ) is more detailed than that in Paper I. Some of the parameters are similar as in Paper I; a temperature power-law index $\beta=-0.37$, density index $\alpha=0.07$, and emissivity factor $f_{0}=0.12$ for the power-law component of the elliptical model with an inner temperature of $700 \mathrm{~K}$ (Paper I, Table 8). However, in our new modeling, the emissivity factor of the inner $1400 \mathrm{~K}$ ring is $0.038 \pm 0.016$ and the radius of the hot $1400 \mathrm{~K}$ ring is $2.29 \pm 0.47 R_{\tau_{\mathrm{K}}}$ or $\sim 0.16 \mathrm{pc}$ (with the above $R_{\tau_{\mathrm{K}}}=0.071 \mathrm{pc}$ ), which is no longer fixed to $1 R_{\tau_{\mathrm{K}}}$ as in Paper I. This $1400 \mathrm{~K}$ ring radius of $\sim 2.29 R_{\tau_{\mathrm{K}}}$, which is relatively large compared to the reverberation radius $R_{\tau_{\mathrm{K}}}$, is a representative thin-ring radius and not the inner radius of an extended ring (see discussion in Sect. 4.1). This large radius probably indicates a relatively shallow, extended innermost dusty structure (Paper I) in NGC 3783.

\section{Conclusion}

We have derived a torus radius of $0.74 \pm 0.23$ mas or $0.16 \pm$ $0.05 \mathrm{pc}$ (thin-ring fit). To derive this NGC 3783 torus radius, we took into account an estimated relative flux contribution of $0.5 \%$ from the underlying host galaxy in the 60 mas AMBER FOV and $21 \%$ from the unresolved accretion disk. This torus radius is approximately 2.3 times larger than the $K$-band reverberation radius $R_{\tau_{\mathrm{K}}} \sim 0.071 \mathrm{pc}$ (see discussion in Sect. 4.1). For the interpretation of the observations, we employed a temperature/density-gradient model including a hot inner $1400 \mathrm{~K}$ ring. We simultaneously fitted our new NIR AMBER visibilities, the MIR MIDI visibilities from Paper I, and the SED to constrain physical parameters of the dust distribution. For the power-law component of the model, we derived a temperature power-law index $\beta \sim-0.37$ and a surface density index $\alpha \sim 0.07$. For the required $1400 \mathrm{~K}$ ring component, a radius of $\sim 2.3$ reverberation radii or $\sim 0.16 \mathrm{pc}$ was found, whereas in the modeling in Paper I, the $1400 \mathrm{~K}$ ring radius was assumed to be one reverberation radius. This $1400 \mathrm{~K}$ ring radius of $\sim 0.16 \mathrm{pc}$, which is relatively large compared to the reverberation radius, is a representative thin-ring radius and not the inner radius of an extended ring. This radius probably indicates a relatively shallow, extended inner dusty structure. Our study of NGC 3783 and the results in Paper I show that the simultaneous modeling of both NIR and MIR interferometric observations is a powerful tool for future detailed studies of AGN tori.

Acknowledgements. We thank the ESO VLTI team on Paranal for the excellent collaboration and the referee for his valuable comments. This publication makes use of the SIMBAD database operated at CDS, Strasbourg, France. Some of the data presented here were reduced using the publicly available data-reduction software package amdlib kindly provided by the Jean-Marie Mariotti Center (http://www.jmmc.fr/data_processing_amber.htm).

\section{References}

Antonucci, R. 1993, ARA\&A, 31, 473

Beckert, T., Driebe, T., Hönig, S. F., \& Weigelt, G. 2008, A\&A, 486, L17

Burtscher, L., Jaffe, W., Raban, D., et al. 2009, ApJ, 705, L53

Burtscher, L., Meisenheimer, K., Jaffe, W., Tristram, K. R. W., \& Röttgering, H. J. A. 2010, PASA, 27, 490

Chelli, A., Utrera, O. H., \& Duvert, G. 2009, A\&A, 502, 705

Glass, I. S. 1992, MNRAS, 256, 23

Hönig, S. F., \& Kishimoto, M. 2010, A\&A, 523, A27

Jaffe, W., Meisenheimer, K., Röttgering, H. J. A., et al. 2004, Nature, 429, 47

Kishimoto, M., Hönig, S. F., Beckert, T., \& Weigelt, G. 2007, A\&A, 476, 713

Kishimoto, M., Hönig, S. F., Antonucci, R., et al. 2009a, A\&A, 507, L57

Kishimoto, M., Hönig, S. F., Tristram, K. R. W., \& Weigelt, G. 2009b, A\&A, 493, L57

Kishimoto, M., Hönig, S. F., Antonucci, R., et al. 2011a, A\&A, 527, A121

Kishimoto, M., Hönig, S. F., Antonucci, R., et al. 2011b, A\&A, 536, A78 (Paper I)

Kreplin, A., Kraus, S., Hofmann, K.-H., et al. 2012, A\&A, 537, A103

Krolik, J. H., \& Begelman, M. C. 1988, ApJ, 329, 702

Meisenheimer, K., Tristram, K. R. W., Jaffe, W., et al. 2007, A\&A, 471, 453

Petrov, R. G., Malbet, F., Weigelt, G., et al. 2007, A\&A, 464, 1

Pott, J.-U., Malkan, M. A., Elitzur, M., et al. 2010, ApJ, 715, 736

Raban, D., Jaffe, W., Röttgering, H., Meisenheimer, K., \& Tristram, K. R. W. 2009, MNRAS, 394, 1325

Suganuma, M., Yoshii, Y., Kobayashi, Y., et al. 2006, ApJ, 639, 46

Swain, M., Vasisht, G., Akeson, R., et al. 2003, ApJ, 596, L163

Tatulli, E., Millour, F., Chelli, A., et al. 2007, A\&A, 464, 29

Tristram, K. R. W., \& Schartmann, M. 2011, A\&A, 531, A99

Tristram, K. R. W., Meisenheimer, K., Jaffe, W., et al. 2007, A\&A, 474, 837

Tristram, K. R. W., Raban, D., Meisenheimer, K., et al. 2009, A\&A, 502, 67

Wittkowski, M., Kervella, P., Arsenault, R., et al. 2004, A\&A, 418, L39 